\title{
Point-contact Andreev-reflection spectroscopy in anisotropic superconductors: the importance of directionality
}

(Review Article)

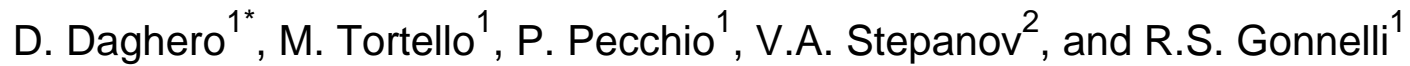 \\ ${ }^{1}$ Dipartimento di Scienza Applicata e Tecnologia, Politecnico di Torino, Torino 10129, Italy \\ E-mail: dario.daghero@polito.it \\ ${ }^{2}$ P.N. Lebedev Physical Institute, Russian Academy of Sciences, Moscow 119991, Russia
}

Received November 1, 2012

\begin{abstract}
Point-contact Andreev-reflection spectroscopy (PCARS) has demonstrated to be one of the most effective experimental tools for the investigation of fundamental properties of superconductors such as the superconducting gap and the electron-phonon (or, more generally, electron-boson) coupling. By reviewing relevant examples reported in literature and presenting new results, in this paper we show that when the direction of the interface with respect to the crystallographic axes can be controlled (as in single crystals and epitaxial films) PCARS can provide invaluable information about the anisotropy of the pairing wavefunction or - in the case of multiband superconductors - on the number, amplitude and symmetry of the energy gaps. Moreover, the analysis of PCARS results within a suitable 3D generalization of the BTK model allows obtaining qualitative information about the topology of the Fermi surface.
\end{abstract}

PACS: 74.45.+c Proximity effects; Andreev effect; SN and SNS junctions;

74.70.-b Superconducting materials other than cuprates;

74.20.Rp Pairing symmetries.

Keywords: point-contact spectroscopy, Andreev reflection, unconventional superconductivity, order-parameter anisotropy.

Contents

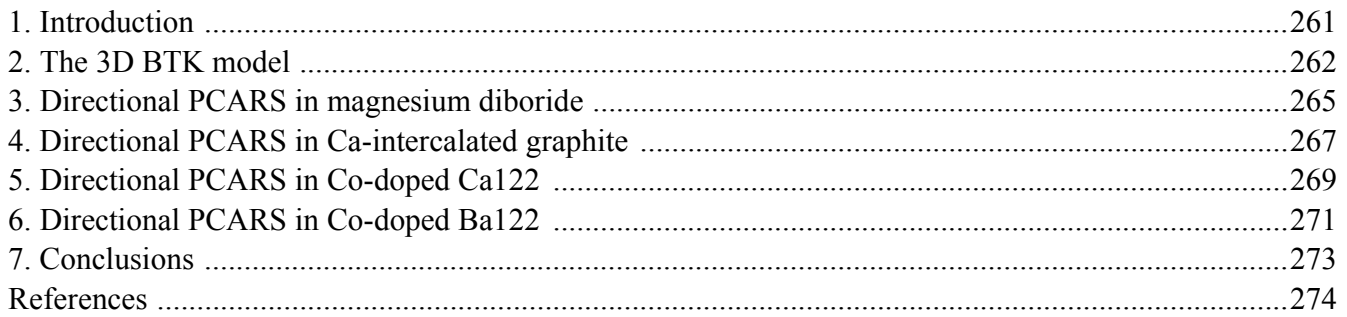

\section{Introduction}

Point-contact spectroscopy (PCS) was developed almost 40 years ago as an experimental tool to investigate the interaction between electrons and phonons in metals. Yanson [1] was the first to realize that some nonlinearities in the $I-V$ characteristics of microscopic constrictions between two metals were the hallmark of inelastic scattering of electrons by phonons. The point-contact technique was later used to study all kinds of scattering of electrons by elementary excitation in metals, like magnons and so on $[2,3]$. When one of the two banks of the point contact is a superconductor, the conduction is dominated by quantum phenomena such as electron tunneling or Andreev reflection depending on the height of the potential barrier at the interface. By solving the Bogoliubov-de Gennes equations near the N/S interface [4] Blonder, Tinkham and Klapwijk (BTK) were able to predict the shape of the point-contact Andreev-reflection spectroscopy (PCARS) curves as a 
function of a dimensionless barrier parameter $Z$ such that $Z=0$ corresponds to a perfectly transparent $\mathrm{N} / \mathrm{S}$ junction, while large values of $Z$ (of the order of 10) correspond to a tunnel junction [5]. The original model was based on a number of simplifying assumptions; in particular, the current injection was assumed to occur along a well-defined direction (normal to the flat N/S interface), and the Fermi velocities in the two banks were assumed to be identical. The first simplifying assumption means that the model was actually 1D and could only describe systems where the Fermi surface in both the banks is spherical and the superconductor is perfectly isotropic (i.e., the order parameter has an $s$-wave symmetry). A generalization of the BTK model to the $2 \mathrm{D}$ case was necessary with the advent of cuprates, that are two-dimensional to a good approximation and show a marked in-plane anisotropy of the pairing wavefunction. In the $2 \mathrm{D}$ version of the BTK model [6] electrons are injected in the whole half-plane, with an angle-dependent probability that also depends on the barrier parameter $Z$. This model was able to explain the observed PCARS spectra in cuprates (and other materials) as being due to an unconventional (in plane) symmetry of the order parameter, e.g., $d$-wave. In particular, it was able to predict the occurrence of zero-bias peaks in the conductance as arising from interference effects between electron-like and hole-like quasiparticles (ELQ and HLQ) that result from the sign change of the order parameter in the $k$ space. The model also removed other simplifying assumptions of the first BTK model (such as, for example, the equality of the Fermi velocities in the two banks) but was still based on the assumption of spherical (circular) Fermi surfaces. The necessity to overcome these limitations became clear with the discovery of $\mathrm{MgB}_{2}$, the first superconductor that unambiguously showed multiple gaps residing on distinct sheets of the Fermi surface with different character: while one is almost cylindrical, the other is a tubular network that is intrinsically $3 \mathrm{D}$ (but not spherical). A simple generalization of the BTK model to this case, which however holds only as long as the gaps are isotropic (i.e., have a s-wave symmetry), was proposed in Ref. 7. Within this model, the normalized conductance of the point contact is simply a weighted sum of the partial contributions of the two bands, the weight of the ith band being proportional to its contribution to the plasma frequency. In the specific case of $\mathrm{MgB}_{2}$, the weight of the $\sigma$-band gap (residing on the quasi2D Fermi surface sheets) was predicted to be as high as $33 \%$ for current injection along the $a b$ plane, and only $1 \%$ for injection along the $c$ axis [7]. The success of the multiband models in describing the physics of $\mathrm{MgB}_{2}$ soon led to the re-analysis of puzzling results obtained in other materials (for example, boron carbides) that, sometimes, were shown to have a multiband character as well (see [8] and references therein). It is worth noting that, in all the multiband materials where different band systems have different dimensionality, directional PCARS measurements become mandatory to attain a good determination of the gap amplitudes. For example, in $\mathrm{MgB}_{2}$ a careful determination of the $\sigma$-band gap necessarily requires injecting the current along the $a b$ planes. The situation became even more complex with the discovery and the investigation of Fe-based superconductors. Although an effective two-band 2D BTK model (analogous to that used in $\mathrm{MgB}_{2}$ ) has been often used (and still is) in the analysis of the PCARS spectra on these materials, it may not always provide accurate information on the symmetry and the amplitudes of the gaps. The compounds of the so-called 1111 family show almost 2D hole-like and electron-like Fermi surface sheets and, in most cases, isotropic order parameters; therefore the aforementioned model can catch the physics of the system, but the relative weight of the two gaps should in principle depend on the direction of current injection. The insufficiency of the effective two-band 2D model becomes clear whenever some anisotropy of the order parameter takes place. This has been predicted to happen in some 1111 compounds [9] and in various 122 compounds [10-12]. The simultaneous presence of Fermi surface sheets with a 3D (but not spherical) character and of (at least one) anisotropic order parameter clearly limits the reliability of a "simple" fit of the PCARS spectra with the model used for $\mathrm{MgB}_{2}$ because it is no longer possible to express the conductance as a weighted sum of the partial contributions of the two sets of bands. Instead a more complicated 3D BTK model that takes into account the shape of the Fermi surface and allows modelling complex 3D anisotropies of the gaps should be used [13]. As we will show, the use of this model gives PCARS unsuspected predictive capabilities concerning the shape of the Fermi surface as well as the presence and the geometry of lines of nodes. In the following of this paper we will present some examples of how directional PCARS has been used and of what kind of information it can provide. Before that, however, we will briefly recall the 3D BTK model.

\section{The 3D BTK model}

In its original formulation, the BTK model [5] was onedimensional, i.e., all the momenta of the incident electrons were assumed to be perpendicular to the N/S interface. It was also based on other simplifying assumptions: i) the Fermi surface is spherical in both banks, i.e., a freeelectron approach is used; ii) the Fermi velocity is the same in both banks; iii) the barrier is ideal and has null thickness, i.e., it is represented by a repulsive potential $U_{0} \delta(x)$ located at the interface. Within these limitations, the normal-state transparency of the barrier $\tau_{N}$ only depends on the dimensionless parameter $Z=U_{0} / \hbar v_{F}$ and contains no geometrical factors: $\tau_{N}=1 /\left(1+Z^{2}\right)$. The point-contact conductance is thus given by 


$$
\sigma(E)=\frac{1+\tau_{N}|\gamma(E)|^{2}+\left(\tau_{N}-1\right)\left|\gamma(E)^{2}\right|^{2}}{\left|1+\left(\tau_{N}-1\right) \gamma(E)^{2}\right|^{2}},
$$

the function $\gamma$ being defined as

$$
\gamma(E)=\frac{(E+i \Gamma)-\sqrt{(E+i \Gamma)^{2}-\Delta^{2}}}{\Delta},
$$

where $\Gamma$ is a broadening parameter [14]. The 1D approach was unable to explain the results of PCARS or tunneling in anisotropic superconductors where the order parameter does not have a $s$-wave symmetry in the $k$ space, as for example in cuprates. In these cases, indeed, it is necessary to account for the fact that electrons can approach the interface from any direction. Calling $\mathbf{n}$ the unit vector normal to the interface and $\theta_{N}$ the angle between $\mathbf{n}$ and the Fermi wavevector of the incident electron, $\mathbf{k}_{F N}$, the normal barrier transparency becomes

$$
\tau_{N}\left(\theta_{N}\right)=\frac{\cos ^{2} \theta_{N}}{\cos ^{2} \theta_{N}+Z^{2}}
$$

Roughly speaking, this means that the probability of electron transmission decreases on increasing $Z$, and for any given $Z \neq 0$ decreases on increasing $\theta_{N}[8,13]$. Now, any incident electron can undergo four possible processes at the interface: a) reflection in $\mathrm{N}$ as a hole (Andreev reflection); b) reflection as electron (normal reflection); c) transmission in S as ELQ; d) transmission in $\mathrm{S}$ as HLQ. For any given wavevector of the incident electron, the problem is $2 \mathrm{D}$ since all the momenta lie on the same plane. However, the wavevectors of ELQ and HLQ in $\mathrm{S}$ make with $\mathbf{n}$ angles $+\theta_{S}$ and $-\theta_{S}$, so that if the order parameter has an in-plane angular dependence on $\theta_{S}$, ELQ and HLQ will feel different (in phase and/or in magnitude) order parameters $\Delta_{+}=\Delta\left(\theta_{S}\right)$ and $\Delta_{-}=\Delta\left(-\theta_{S}\right)$ [6]. As a result the relative transparency of the junction in the superconducting state becomes [6]

$$
\sigma_{S}\left(E, \theta_{N}\right)=\frac{1+\tau_{N}\left(\theta_{N}\right)\left|\gamma_{+}\left(E, \theta_{N}\right)\right|^{2}+\left(\tau_{N}\left(\theta_{N}\right)-1\right)\left|\gamma_{+}\left(E, \theta_{N}\right) \gamma_{-}\left(E, \theta_{N}\right)\right|^{2}}{\left|1+\left(\tau_{N}\left(\theta_{N}\right)-1\right) \gamma_{+}\left(E, \theta_{N}\right) \gamma_{-}\left(E, \theta_{N}\right) \exp \left(i \varphi_{d}\left(\theta_{N}\right)\right)\right|^{2}},
$$

where

$$
\gamma_{ \pm}\left(E, \theta_{N}\right)=\frac{(E+i \Gamma)-\sqrt{(E+i \Gamma)^{2}-\left|\Delta_{ \pm}\right|^{2}}}{\left|\Delta_{ \pm}\right|}
$$

and

$$
\varphi_{d}\left(\theta_{N}\right)=-i \ln \left[\frac{\Delta_{+} /\left|\Delta_{+}\right|}{\Delta_{-} /\left|\Delta_{-}\right|}\right]
$$

is the phase difference seen by the HLQ with respect to the ELQ. Note that in Eqs. (5) and (6) the dependence on $\theta_{N}$ results from the dependence of $\Delta$ on $\theta_{S}$ and from the fact that $k_{F N} \sin \left(\theta_{N}\right)=k_{F S} \sin \left(\theta_{S}\right)$ as required by the conservation of the momenta in the direction parallel to the interface. It is worthwhile to remark that here we have assumed an angular dependence of the order parameter on the plane defined by the wavevector of the incident electron and by $\mathbf{n}$. This corresponds, for example, to PCARS experiments in cuprates with the current injected along the $a b$ plane. The normalized conductance that can be compared to (normalized) PCARS spectra is finally given by

$$
G_{2 \mathrm{D}}(E)=\frac{\int_{-\pi / 2}^{+\pi / 2} \sigma_{S}(E, \theta) \tau_{N}(\theta) \cos \theta d \theta}{\int_{-\pi / 2}^{+\pi / 2} \tau_{N}(\theta) \cos \theta d \theta} .
$$

This model can be generalized in a straightforward manner to the case of multiband superconductors, as shown in [8] and [13], by simply expressing the total normalized conductance as the weighted sum of the partial contributions of each band. The fitting function will contain various parameters: in addition to $\Delta$ (maximum amplitude of the gap), $\Gamma$ (broadening) and $Z$ for each band, there will be the weight of the contributions of the various bands. With two bands, the parameters are already seven; the use of more bands risks to make the fit meaningless. Indeed, in all the cases of multiband superconductors studied so far (e.g., $\mathrm{MgB}_{2}$, borocarbides, Fe-based superconductors) effective two-band models have been proposed by treating similar bands on the same footing.

As already pointed out, the model described so far works well in isotropic superconductors or layered superconductors, like cuprates, provided that the current is injected along the $a b$ plane. One of the basic assumptions it is based on, however, is that the Fermi surface is spherical (circular) in both the $\mathrm{N}$ and the $\mathrm{S}$ side (indeed, the magnitudes of the Fermi wavevectors $k_{F N}$ and $k_{F S}$ can be different but do not depend on the angle). If the Fermi surfaces were really spherical, a generalization to the $3 \mathrm{D}$ case could be achieved easily by integrating over the whole solid angle, eventually including the full dependence of the order parameter on the azimuthal and inclination angles [6]. The problem remains of how to express the $c$-axis conductance in cuprates (where the Fermi surface is practically cylindrical) and, more generally, the conductance in multiband superconductors with complex shapes of the Fermi surface. 
A generalization of the model to a 3D case with arbitrarily-shaped Fermi surface sheets is conceptually easy, though it can become very demanding from the point of view of calculations. The general equation that provides the normalized conductance at $T=0$ was given in Ref. 8 and reads

$$
\langle G(E)\rangle_{I \| \mathbf{n}}=\frac{\sum_{i}\left\langle\sigma_{i \mathbf{k} n}(E) \tau_{i \mathbf{k}, n} \frac{v_{i \mathbf{k}, n}}{v_{i \mathbf{k}}}\right\rangle_{F S_{i}}}{\sum_{i}\left\langle\tau_{i \mathbf{k}, n} \frac{v_{i \mathbf{k}, n}}{v_{i \mathbf{k}}}\right\rangle_{F S_{i}}} .
$$

In this equation, $i$ is the band index, the brackets indicate an average over the $i$ th Fermi surface sheet, the subscript $n$ refers to the direction of current injection, $v_{i \mathbf{k}, n}=\mathbf{v}_{i \mathbf{k}} \cdot \mathbf{n}$ is the projection of the velocity on the $i$ th band along the direction of the unit vector $\mathbf{n}$. The normal-state barrier transparency is

$$
\tau_{i \mathbf{k}, n}=\frac{4 v_{i \mathbf{k}, n} v_{N, n}}{\left(v_{i \mathbf{k}, n}+v_{N, n}\right)^{2}+4 Z^{2} v_{N}^{2}}
$$

where $v_{N, n}=\mathbf{v}_{N} \cdot \mathbf{n}, \quad \mathbf{v}_{N}$ being the (constant in magnitude) Fermi velocity in the normal material. In Eq. (8) the quantity $\sigma_{i \mathbf{k} n}(E)$ is expressed by an equation similar to (4), but where $\tau_{N}\left(\theta_{N}\right) \rightarrow \tau_{i \mathbf{k}, n}$, and the order parameter in the $i$ th band is dependent on $\mathbf{k}$, i.e., $\Delta_{i}(\mathbf{k})=\Delta_{i, \mathbf{k}}$.

The calculation is greatly simplified in particular cases. For example, if the barrier is very high $(Z \rightarrow \infty)$

$$
\langle G(E)\rangle_{I \| \mathbf{n}} \cong \frac{\sum_{i}\left\langle\sigma_{i \mathbf{k} n}(E) \frac{v_{i \mathbf{k}, n}^{2}}{v_{i \mathbf{k}}}\right\rangle_{F S_{i}}}{\sum_{i}\left\langle\frac{v_{i \mathbf{k}, n}^{2}}{v_{i \mathbf{k}}}\right\rangle_{F S_{i}}}=\frac{\sum_{i}\left\langle\sigma_{i \mathbf{k} n}(E) D_{i \mathbf{k}} v_{i \mathbf{k}, n}^{2}\right\rangle_{F S_{i}}}{\sum_{i}\left\langle D_{i \mathbf{k}} v_{i \mathbf{k}, n}^{2}\right\rangle_{F S_{i}}}
$$

where $D_{i \mathbf{k}}=1 /\left\{4 \pi^{3}\left|\nabla_{\mathbf{k}}\left[E_{i}(\mathbf{k})\right]\right|\right\}_{E_{F}}$ is the normal density of states of the $i$ th band at the Fermi energy and wave vector $\mathbf{k}$ in $\mathrm{S}$. In general, $\sigma_{i \mathbf{k} n}(E)$ cannot be taken outside the integral because it contains $k$-dependent quantities like $\Delta_{i}$ and $\tau_{i \mathbf{k}, n}$ (see Eq. (4)) and thus $v_{i \mathbf{k}, n}$ (see Eq. (9)). However, if $Z \rightarrow \infty$ and if the order parameters are isotropic one can approximate the problem by assuming that $\sigma_{i \mathbf{k} n}(E)=\sigma_{i}(E)$, i.e., it does not have any dependence on k. Note that this implies using the 1D BTK model (Eq. (1)) to calculate $\sigma_{i}(E)$. In these conditions, the normalized conductance simply becomes a weighted average of the contributions of the various bands, where the weights are often briefly indicated in literature as $\left\langle N v^{2}\right\rangle[15]$. By the way, this approach has been used (as we will show later) to predict the results of directional PCARS in the case of $\mathrm{MgB}_{2}$ [7].

Another case in which Eq. (8) acquires a more appealing form is when $Z=0$, the Fermi surface is spherical and the order parameters are all isotropic. In this case, $\tau_{i \mathbf{k}, n}$ is constant and the partial conductances lose any dependence on $\mathbf{k}$ (i.e., $\left.\sigma_{i \mathbf{k}, n}(E)=\sigma_{i}(E)\right)$ and can thus be taken outside the integral over the Fermi surfaces. In these conditions, Eq. (8) reduces again to a weighted average of the partial conductances of the bands where the weights can be expressed as $\left\langle D_{i \mathbf{k}} v_{i \mathbf{k}, n}\right\rangle_{F S_{i}}[15]$ :

$$
\langle G(E)\rangle_{I \| \mathbf{n}}=\frac{\sum_{i} \sigma_{i}(E)\left\langle D_{i \mathbf{k}} v_{i \mathbf{k}, n}\right\rangle_{F S_{i}}}{\sum_{i}\left\langle D_{i \mathbf{k}} v_{i \mathbf{k}, n}\right\rangle_{F S_{i}}}=\frac{\sum_{i} \sigma_{i}(E) S_{i, n}}{\sum_{i} S_{i, n}}
$$

where $S_{i, n}$ is the area of the projection of the $i$ th sheet of the Fermi surface on a plane perpendicular to $\mathbf{n}$. In this very particular and simplified case, therefore, an immediate geometrical interpretation of the normalized conductance can be given, that immediately clarifies the importance of the dimensionality of the Fermi surface sheets in determining the point-contact Andreev-reflection spectra.

In the general case of intermediate barrier height and anisotropic gaps, neither Eq. (10) nor Eq. (11) can be used to calculate the PCARS spectra. Equation (8) and the real shape of the Fermi surface must be used instead. The conductance of the N/S junction in general cannot be expressed as a weighted average of the partial contributions of the various bands, but one can still talk of the "weight" of the $i$ th band referring to the normal state conductance (when $\sigma_{i \mathbf{k}, n}=1$ ). This "weight" is determined solely by the normal-state barrier transparencies $\tau_{i \mathbf{k}, n}$, containing the barrier parameters $Z_{i}$ (see Eq. (9)), and by the shape of the Fermi surface sheets.

It is clear from the above that the calculation of the normalized PCARS spectra in the most general case is a feasible task, but can be rather tough. If one has access to the explicit full $\mathbf{k}$ dependence of the order parameter, or better the map of the gap over the Fermi surface, then Eq. (8) can be used to predict the expected shape of the PCARS spectra for arbitrary directions of current injections, and with a given value of the barrier parameter(s). These curves can then be compared to the experimental ones to determine whether the theoretical $\Delta_{i}(\mathbf{k})$ is plausible or not.

Often, however, one performs PCARS experiments with little or no suggestion about what the symmetry of the order parameter could be. In these cases, the form of the $\Delta_{i}$ (k) functions that allows best fitting or at least qualitatively reproducing the experimental spectra must be found. This procedure would require repeating many times the calculations of Eq. (8) by slightly changing the adjustable parameters and this can be too time-consuming if the real Fermi surface is used. A compromise between the necessities of a fast calculation and of a greater accuracy of the fitting model consists: i) in using a model Fermi surface made up of analytical surfaces (spheroids, hyperboloids and so on) that mimic as well as possible the "real" ones; and ii) in making a simple guess about the symmetry of the 
order parameter by using analytical expressions of the $\Delta_{i}(\mathbf{k})$ functions. In this way, the complex integral over the Fermi surface can be reduced to easily solvable integrals over the spherical coordinates (in the reciprocal space) $k_{F}$ (magnitude of the Fermi wavevector), $\theta$ (azimuthal angle) and $\phi$ (inclination angle). The explicit expressions of Eq. (8) for different directions of current injection are reported elsewhere [13]. In the following sections, we will show how this 3D BTK model allows reproducing the shape of the PCARS spectra in various multiband superconductors like $\mathrm{MgB}_{2}, \mathrm{CaC}_{6}$ and Fe-based superconductors of the 122 family.

\section{Directional PCARS in magnesium diboride}

$\mathrm{MgB}_{2}$ has a layered structure with graphite-like, honeycomb $\mathrm{B}$ layers intercalated by $\mathrm{Mg}$ planes with hexagonal close-packed structure. Its Fermi surface, shown in

\author{
(a)
}

(d)

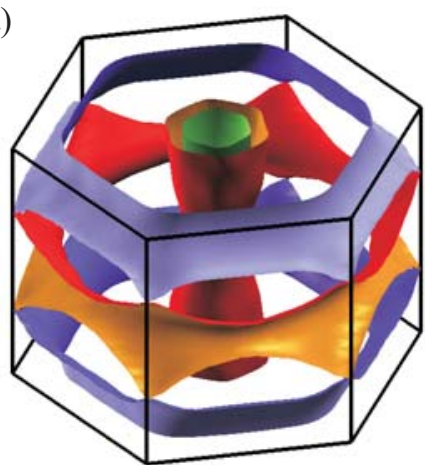

Experimental spectra
Fig. 1(a), is made up of two hole-like warped cylinders (originated by the $\sigma$ bands) whose axis lies on the $\Gamma-\mathrm{A}$ line, and of a 3D electron-like tubular network (originated by the $\pi$ bands) [16]. It was soon understood that this structure allowed the coexistence of two distinct superconducting energy gaps [17], and indeed a number of experimental tools gave anomalous results that could find an explanation only within a two-gap picture.

Paradoxically, the first point-contact and tunnel spectroscopy measurements in polycrystalline samples gave contradicting results, sometimes showing two gaps, sometimes only one [18]. The explanation of this issue required some more theoretical investigation. In Ref. 7 the problem of understanding how multiband superconductivity manifests itself in tunneling measurements (large Z) was solved by using Eq. (10) and further simplifying the problem thanks to the fact that the gaps are isotropic (in practice, a 1D injection was assumed). In the end, the normalized

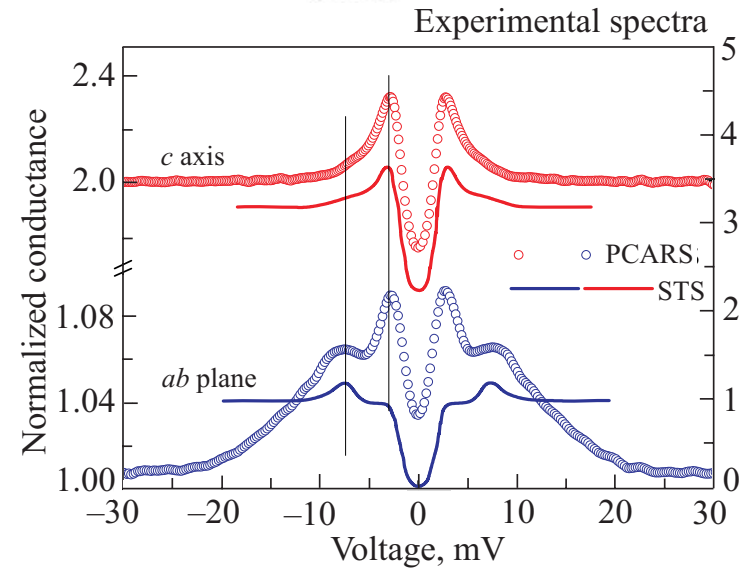

(b)

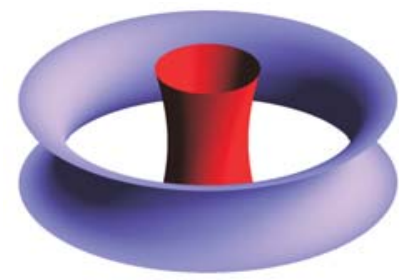

(c)

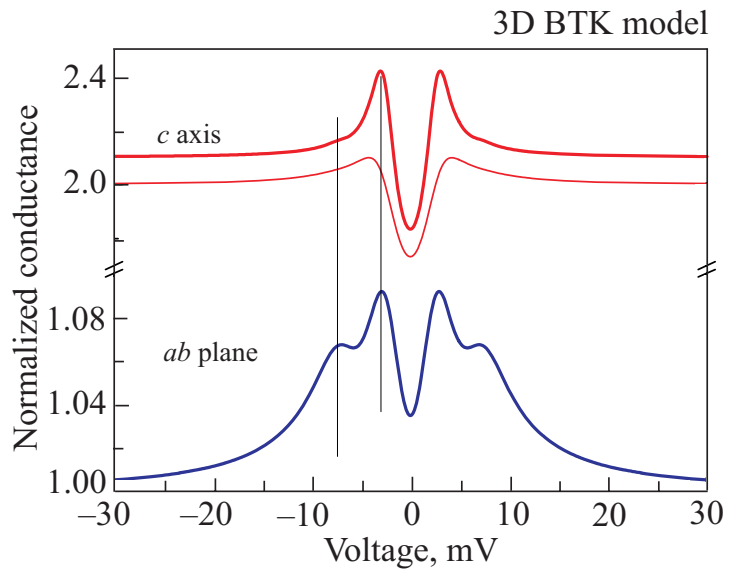

Fig. 1. (Color online) (a) Fermi surface of $\mathrm{MgB}_{2}$. (b) Model Fermi surface used for the calculation of the theoretical spectra within the two-band 3D BTK model. (c) Theoretical PCARS curves generated at $T=4.2 \mathrm{~K}$ by using the two-band 3D BTK model with the gaps calculated in Ref. 7, i.e., $\Delta_{\pi}=2.70 \mathrm{meV}$ and $\Delta_{\sigma}=7.09 \mathrm{meV}$ and the model Fermi surface depicted in (b), for $I \| a b$ (bottom) and $I \| c$ (top). The bottom (blue) and the top thin (red) curves were obtained by using the same parameters, i.e., $Z_{\pi}=0.37, \Gamma_{\pi}=1.65 \mathrm{meV}$, $Z_{\sigma}=0.85, \Gamma_{\sigma}=2.10 \mathrm{meV}$, that allow reproducing the $a b$-plane spectrum of panel (d); the top thick (red) curve has parameters optimized to reproduce the shape of the $c$ axis spectrum of panel (d), i.e. $Z_{\pi}=0.17, \Gamma_{\pi}=0.5 \mathrm{meV}, Z_{\sigma}=0.85, \Gamma_{\sigma}=0.4 \mathrm{meV}$. (d) Symbols: two examples of PCARS spectra taken in $\mathrm{MgB}_{2}$ single crystals, with current injected along the $a b$ plane (bottom) and along the $c$ axis (top). The vertical scale is on the left axis; the top curve has been offset by 1 for clarity. Lines: two examples of STS spectra taken in different grains of a polycrystal (from Ref. 24). The vertical scale is on the right axis and the top curve has been offset for clarity. Vertical lines show the correspondence of the gap features in PCARS and STS spectra along the different directions. 
conductance was expressed as a weighted average of the contribution from the $\sigma$ and $\pi$ bands, with weighting factors of the form $\left\langle D_{i \mathbf{k}} v_{i \mathbf{k}, n}^{2}\right\rangle \propto\left(\omega_{\mathrm{pl}}^{i}\right)^{2}$ where $\omega_{\mathrm{pl}}^{i}$ is the contribution of the electrons in the ith band to the plasma frequency. In this limit, the ratio of the normal state conductivities is equal to the ratio of the square of the plasma frequencies and the total normalized conductance of a SIN junction can be expressed as

$$
\begin{aligned}
G(V) & =\frac{\left(\omega_{\mathrm{pl}}^{\pi}\right)^{2} \sigma_{\pi}(V)+\left(\omega_{\mathrm{pl}}^{\sigma}\right)^{2} \sigma_{\sigma}(V)}{\left(\omega_{\mathrm{pl}}^{\pi}\right)^{2}+\left(\omega_{\mathrm{pl}}^{\sigma}\right)^{2}}= \\
& =w_{\pi} \sigma_{\pi}(V)+\left(1-w_{\pi}\right) \sigma_{\sigma}(V)
\end{aligned}
$$

where the partial normalized conductances of the two bands $\sigma_{\pi, \sigma}(V)$ can be calculated within the BTK model. Despite the rather crude approximations of the derivation, the model is able to explain the apparent contradictions in the number of gaps observed by the first PCARS measurements in polycrystals as being due to the large anisotropy of the plasma frequencies. Indeed, the predicted relative weight of the $\sigma$ band is very small for tunneling in the $c$ direction (of the order of $1 \%$ ) so that if the current is injected along the $c$ axis of a given grain only the smaller $\pi$-band gap is observed. Instead, for tunneling along the $a b$ planes both the gaps are clearly visible. It is worthwhile to note that the discernibility of the two gaps is made possible by the large difference in their amplitudes. A map of the gap distribution over the Fermi surface obtained by Choi et al. [19] shows that the gap amplitudes are to a good extent uniform over the cylindrical and the tubular surfaces: the distributions of the $\sigma$ and $\pi$ gaps are rather narrow and do not overlap so that it is reasonable to associate uniform gaps to each surface.

The (uniform over the relevant FS sheet) values of the gaps were calculated by Brinkman et al. [7] within the Eliashberg theory giving $\Delta_{\pi}=2.70 \mathrm{meV}$ and $\Delta_{\sigma}=$ $=7.09 \mathrm{meV}$. Indeed, these values are very similar to those $\left(\Delta_{\pi}=2.8 \mathrm{meV}\right.$ and $\left.\Delta_{\sigma} \simeq 7.0 \mathrm{meV}\right)$ that Szabó et al. [20] had already obtained by fitting the PCARS spectra measured in different regions of a polycrystal with an effective two-band BTK model where the conductance was expressed as $\alpha \sigma_{\pi}+(1-\alpha) \sigma_{\sigma}$, with the weight $\alpha$ being an adjustable parameter ranging between 0.1 and 0.9. Similar results were obtained a few months later in thin films of $\mathrm{MgB}_{2}$ [21], where the fit of experimental PCARS spectra with an adjustable weight gave $\Delta_{\pi}=(2.3 \pm 0.3) \mathrm{meV}$ and $\Delta_{\sigma}=(6.2 \pm 0.7) \mathrm{meV}$. By carrying out a large number of PCARS measurements in oriented $\mathrm{MgB}_{2}$ films and fitting the spectra with an effective two-band model with adjustable weight, Yanson and coworkers determined the experimental distribution of gap amplitudes [22,23]. As for the small gap, this distribution is much narrower than the theoretical one calculated by Choi et al. [19], and is peaked at
$\Delta_{\pi}=2.45 \mathrm{meV}$; the distribution of the large gap has a width similar to the theoretical one, but has a single maximum at $\Delta_{\sigma}=7.0 \mathrm{meV}$. Scanning tunneling microscopy measurements on single grains of a polycrystals [24] gave clear evidence of grain-dependent (i.e., direction-dependent) spectra that were fitted to an effective two-band BTK model, giving the gap amplitudes $\Delta_{\pi}=2.3 \mathrm{meV}$ and $\Delta_{\sigma}=7.1 \mathrm{meV}$. The weight of the $\pi$-band contribution was determined from the fit and turned out to range from $100 \%$ to about $70 \%$ depending on the grain, in good agreement with predictions.

It was only with the advent of good-quality single crystals (which allowed controlling the direction of the injected current and/or of the applied magnetic field with respect to the crystallographic axes) that the potential of PCARS could be exploited to its best. For example, thanks to directional point-contact measurements in single crystals (in the inverse configuration, i.e., using a $\mathrm{MgB}_{2}$ crystal as the "needle") in magnetic fields parallel to the $c$ axis, Yanson's group was the first to resolve the so-called $E_{2 g}$ phonon mode in the $d^{2} V / d I^{2}$ spectra [25] thus proving that this phonon mode is coupled to $\sigma$-band carriers and is thus responsible for the superconducting pairing [26]. Moreover, PCARS in single crystals allowed testing the predictions about the weights of the two band systems in the tunnel conductance. Actually, since the crystals grow in the form of thin platelets with the normal parallel to the $c$ axis, making point contacts in the $I \| a b$ configuration by using the standard needle-anvil configuration was almost impossible. Therefore we used a "soft" PCARS technique in which a small drop of Ag conductive paste put on the sample surface plays the role of the normal-metal electrode. This technique allows placing the contact anywhere on the surface, included the side faces, so as to inject the current either parallel or perpendicular to the $a b$ planes. Figure 1(d) presents two examples of spectra measured by us in $\mathrm{MgB}_{2}$ single crystals, with $I \| a b$ (bottom) and $I \| c$ (top) [27]. For comparison, the spectra taken by scanning tunneling spectroscopy (STS) in single grains of a polycrystals (from Ref. 24) are also shown. It is clear that, while for $I \| a b$ two pairs of peaks symmetric with respect to zero bias are observed, in the $I \| c$ configuration only the peaks associated to the small gap are clearly observed, while the large gap manifests itself only in a pair of shoulders. A fit to the experimental PCARS curves with an effective two-band model, i.e., $G(V)=w_{\pi} \sigma_{\pi}+\left(1-w_{\pi}\right) \sigma_{\sigma}$ (where $\sigma_{\pi, \sigma}$ are calculated within the 2D BTK model and the weight is considered as an adjustable parameter) gives: for $I \| a b, \quad \Delta_{\pi}=2.8 \mathrm{meV}, \Delta_{\sigma}=7.2 \mathrm{meV}$ and $w_{\pi}=0.75$; for $I \| c, \Delta_{\pi}=2.53 \mathrm{meV}, \Delta_{\sigma}=6.8 \mathrm{meV}$ and $w_{\pi}=0.96$. It is clear that the best fit is not obtained by using the weight predicted in the tunneling case [7]; the discrepancy is very small for injection along the $c$ axis, but is larger in the $a b$-plane contacts. The reason is obviously that the proportionality between the weight and the 
plasma frequency, which is at the base of the two-band model for tunneling by Brinkman et al. is true only when $Z \rightarrow \infty$ and for one-dimensional injection, which is not the case here since the values of the barrier parameters turn out to be: $Z_{\pi}=0.484$ and $Z_{\sigma}=1.03$ for $I \| a b, Z_{\pi}=0.775$ and $Z_{\sigma}=0.2$ for $I \| c$. As for the gaps, the values reported above refer to these spectra only, but averaging over different spectra [27] we were able to evaluate the range of variation of the gaps obtaining $\Delta_{\sigma}=(7.1 \pm 0.5) \mathrm{meV}$ and $\Delta_{\pi}=(2.9 \pm 0.3) \mathrm{meV}$, which are in excellent agreement with the predictions.

Now a step forward in the analysis of the PCARS spectra in $\mathrm{MgB}_{2}$ can be made by using the 3D BTK model to see whether, and to which extent, its results differ from those of the simplified two-band BTK model in the tunneling regime, particularly concerning the "weight" of the partial $\pi$ and $\sigma$ contributions. The calculation can be simplified by using, instead of the real Fermi surface, an effective "model FS" that has already been used in the past [28]. The simplification is based on the assumption that the two tubular networks and the two warped cylinders are degenerate (this is equivalent to generally speaking of $\sigma$ and $\pi$ bands without making any distinction within the two systems). As shown in Fig. 1(b), this model FS consists of a warped cylinder centered at the $\Gamma$ point of the BZ and of a half ring torus that simulates the $3 \mathrm{D}$ tubular network associated to the $\pi$ bands. The major and minor radii $\left(R_{\pi}\right.$ and $r_{\pi}$ ) of the torus should be chosen in such a way to reproduce the real Fermi surface as accurately as possible [28]. Once the Fermi surface is known, the calculation of the normalized conductance can be performed by using the explicit version of Eq. (8) for the two different directions as reported in Ref. 13. Figure 1(c) shows the results of this calculation. We took $\Delta_{\pi}=2.70 \mathrm{meV}, \Delta_{\sigma}=7.09 \mathrm{meV}$, as calculated in Ref. 7, and adjusted the barrier parameters $Z_{\pi}$ and $Z_{\sigma}$ in order to reproduce the general shape of the experimental curves of Fig. 1(d). Actually, for this purpose we had also to introduce in the 3D BTK model broadening parameters $\Gamma_{\sigma}$ and $\Gamma_{\pi}$, and perform the calculation at the experimental temperature of $4.2 \mathrm{~K}$. The resulting curves are shown in Fig. 1(c) as solid lines. The bottom line refers to the $a b$-plane contact and was obtained by using $Z_{\pi}=0.37, \Gamma_{\pi}=1.65 \mathrm{meV}, Z_{\sigma}=0.85, \Gamma_{\sigma}=2.10 \mathrm{meV}$. The "weight" of the $\pi$ band (in the sense defined above) is equal to 0.88 and is thus much higher than that predicted in the case of unidirectional current injection and $Z \rightarrow \infty$ [7] (note that also in the 2D BTK fit the weight of the $\pi$ band had to be taken of the order of $75 \%$ ). By using the same parameters, but with the current injection along the $c$ axis, we obtained the thinner of the two top curves of Fig. 1(c). In this case, the weight of the $\pi$ band is as high as 0.98 , in better agreement with the values calculated in the 1D tunneling limit [7]. To obtain a good fit (thick line) of the experimental $c$-axis spectrum, however, we had to reduce both the broadening parameters and change $Z_{\pi}$ (i.e., we had to use $Z_{\pi}=0.17, \Gamma_{\pi}=0.5 \mathrm{meV}$ and $\Gamma_{\sigma}=0.4 \mathrm{meV}$ ) but the weight remains practically the same. Note that the reduction in $\Gamma_{\sigma, \pi}$ is necessary because the amplitude of the Andreev signal in the $c$-axis curve of Fig. 1(d) is higher than in the $a b$-plane one. The reduction in $Z_{\pi}$ is necessary because of the "Z-enhancing effect" described elsewhere [13], i.e., a depletion of the zero-bias conductance that arises from the shape of the Fermi surface and is particularly evident when large portions of it are parallel to $\mathbf{n}$. This effects generally makes the spectra feature zero-bias minima even if the barrier parameter is very small or zero.

\section{Directional PCARS in Ca-intercalated graphite}

$\mathrm{CaC}_{6}$ shows the highest $T_{c}=11.5 \mathrm{~K}$ among graphiteintercalated compounds. Its lattice structure is similar to that of $\mathrm{MgB}_{2}$ in the sense that it shows alternating graphite layers (with honeycomb structure) and Ca planes, with a rhombohedral structure [29]. Indeed, also the bandstructure is similar to that of magnesium diboride and includes both the $\sigma$ and $\pi$ bands. The difference, however, is that in this case the former are completely filled and play no role in superconductivity [30]. Moreover, there is a threedimensional, nearly free-electron band, sometimes called interlayer band, that crosses the Fermi level. This band is also present, although unoccupied, in pure graphite, but in $\mathrm{CaC}_{6}$ it is formed by both $\mathrm{Ca}$ and $\mathrm{C}$ orbitals [30]. As suggested by bandstructure calculations $[31,32]$ it has a fundamental role in superconductivity, because its carriers are coupled to both $\mathrm{Ca}$ and $\mathrm{C}$ phonon modes.

The Fermi surface of $\mathrm{CaC}_{6}$ is shown in Fig. 2(a). It is made up of a series of warped cylindrical surfaces parallel to the $k_{z}$ direction. One of these intersects the nearly-spherical Ca orbital [33]. As pointed out in Refs. 30 and 33 this prevents any separation between Fermi surface sheets associated with the interlayer band and sheets associated with the $\mathrm{C}$ bands, i.e., the nature of electron states changes in the different sheets but also within each sheet. $\mathrm{CaC}_{6}$ cannot thus be considered a multiband superconductor, but a k-dependence of the superconducting order parameter is expected and was indeed predicted by ab-initio calculations within the superconducting density functional theory (SCDFT) [33]. A map of the gap amplitude on the Fermi surface [33] shows that the gap is on average smaller on the warped cylinders and larger on the sphere - actually, the relevant distributions of gap values do not overlap except in the region of intersection of the FS sheets. Owing to the different dimensionality of these Fermi surface sheets it is reasonable to expect that directional PCARS measurements can unveil some anisotropy in the amplitude of the gap, depending on whether the current is injected parallel or perpendicular to the $c$ axis. 

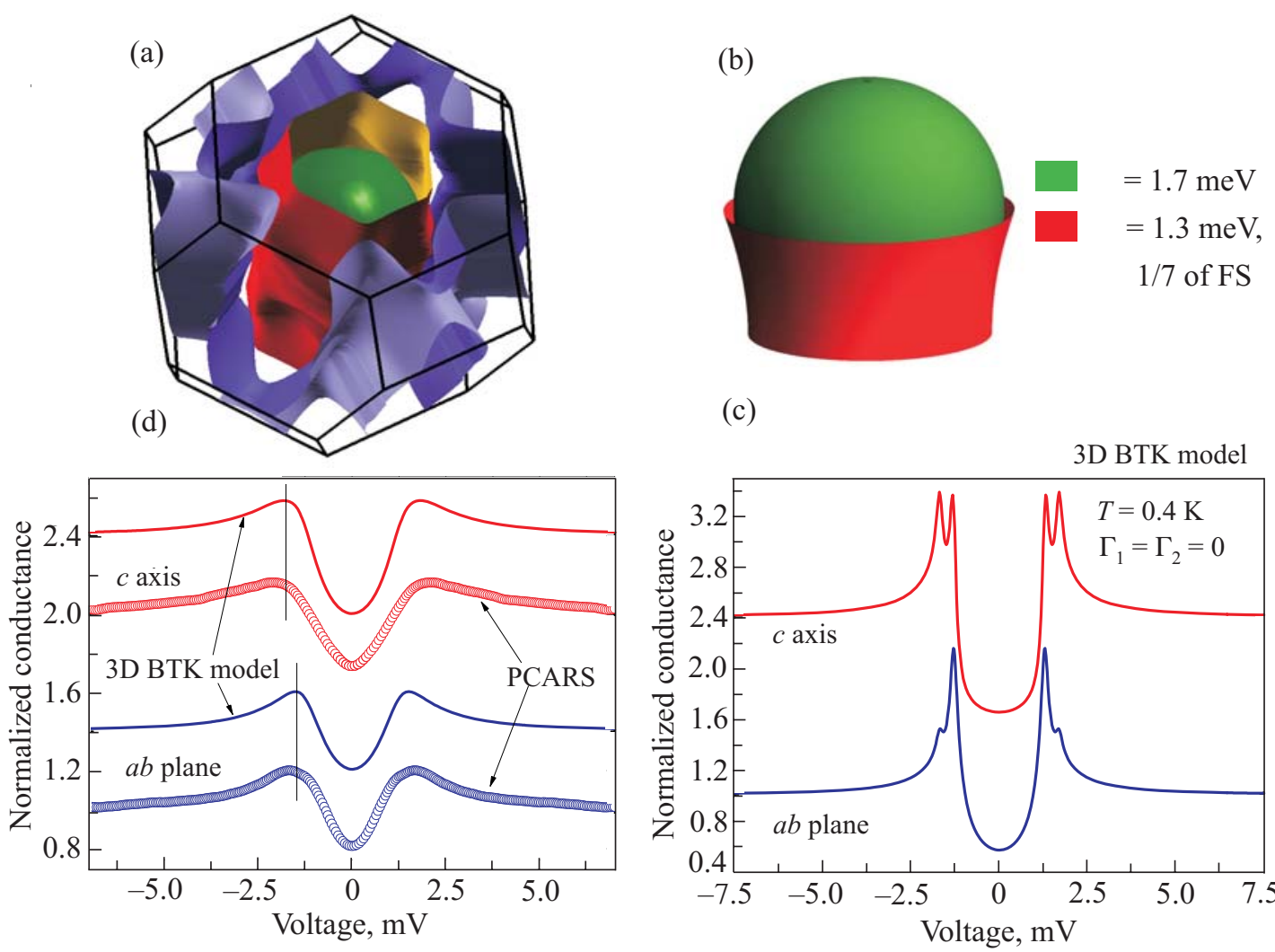

(c)

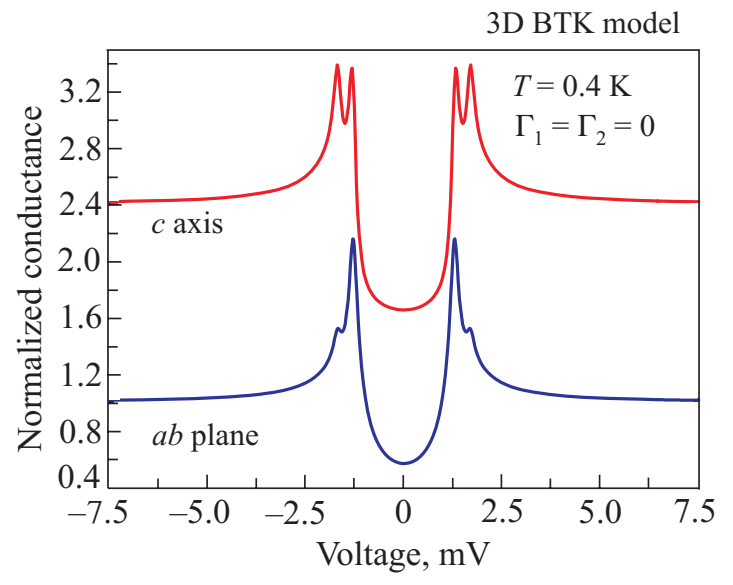

Fig. 2. (a) Fermi surface of $\mathrm{CaC}_{6}$. The almost spherical surface originates from the nearly-free electron band (interlayer band) while the warped cylinders are mainly arising from the carbon $\pi$ bands. As shown in [30], this distinction is however not strict. (b) Model Fermi surface used for the calculations within the 3D BTK model. For simplicity, we assumed two isotropic gaps of different amplitudes (indicated by different colors) to reside on the spherical surface and on the warped cylinders (here represented by a hyperboloid of revolution). Note that in the cell of panel (a) there are 7 cylinders for each spherical surface. (c) Theoretical spectra at $T=400 \mathrm{mK}$ calculated by using the 3D BTK model and the schematic Fermi surface of panel (b). Apart from the gap values $\Delta_{1}=1.7 \mathrm{meV}$ and $\Delta_{2}=1.3 \mathrm{meV}$ that reside on the two portions of the FS, the parameters $Z_{1}=0.60$ and $Z_{2}=0.95$ were used. Note that these values are the same for both $I \| c$ and $I \| a b$. Finally, the broadening parameters were set to zero. (d) Symbols: experimental PCARS spectra measured at $400 \mathrm{mK}$ with current injected along the $c$ axis (top) and along the $a b$ plane (bottom). Thin lines are the theoretical spectra calculated as in panel (c) but using the broadening parameters $\Gamma_{1}=0.8 \mathrm{meV}$ and $\Gamma_{2}=0.5 \mathrm{meV}$.

The first STS results in $\mathrm{CaC}_{6}$ showed a single and apparently isotropic gap but gave conflicting results concerning its amplitude: from 1.6 [34] to $2.3 \mathrm{meV}$ [35], although all these experiments were carried out, at least nominally, with the current injected along the same direction ( $c$ axis). To address the issue of the gap amplitude and of its possible anisotropy, we performed directional PCARS experiments in $\mathrm{CaC}_{6}$ single crystals [36].

Figure 2(d) shows two examples of PCARS curves (symbols) measured at $0.4 \mathrm{~K}$ with current either along the $a b$ plane (bottom) or along the $c$ axis (top). Unlike in $\mathrm{MgB}_{2}$, the curves show one single pair of symmetric peaks in either direction. Moreover, it is possible to fit each of them (as well as all the others we measured) with the 2D single-band BTK model by using an s-wave gap. However, as clearly shown by the position of the peaks, the amplitude of the gap is not the same along the two directions. As for the curves shown in Fig. 2(d), the fit with the 2D BTK mo- del was obtained by using $\Delta=1.44 \mathrm{meV}, \Gamma=0.61 \mathrm{meV}$ and $Z=0.75$ for the $a b$-plane contact, $\Delta=1.7 \mathrm{meV}, \Gamma=$ $=0.84 \mathrm{meV}$ and $Z=0.97$ for the $c$-axis contact. The gap values correspond to values of $2 \Delta / k_{B} T_{C}$ equal to 2.98 and 3.48 , respectively. The reproducibility of these results was very high. In $15 a b$-plane measurements carried out at 0.4 and $4.2 \mathrm{~K}$ the order parameter $\Delta$ ranged between 1.1 and $1.7 \mathrm{meV}$ following rather well a Gaussian distribution peaked at $\overline{\Delta_{a b}}=1.35 \mathrm{meV}$ and with standard deviation $s_{a b}=0.14 \mathrm{meV}$. In $14 \mathrm{c}$-axis contacts the gap ranged between 1.3 and $1.94 \mathrm{meV}$ with a Gaussian distribution with mean $\overline{\Delta_{C}}=1.71 \mathrm{meV}$ and standard deviation $s_{C}=0.08 \mathrm{meV}$ (in good agreement with the values reported in Ref. 34). For the sake of the following discussion, it is very interesting to note that the $Z$ values observed in $c$-axis contacts (between 0.74 and 1.01) are systematically greater than those of $a b$ plane contacts (between 0.48 and 0.75 ). 
In Ref. 36 we also showed that the difference in the spectra taken along the $a b$ plane and along the $c$ axis is perfectly compatible with the distribution of gap values calculated from first principles [33] within the SCDFT. There, we used the full $\mathbf{k}$ dependence of the order parameter and calculated the spectra (at $T=0$ ) by using the exact integration over the Fermi surface according to Eq. (8). Here we show that a very good approximation of the complete calculation, and a good agreement with the experiment, can be obtained by using an effective two-band 3D BTK model based on a model Fermi surface that disregards the most complicated and fine details of the real Fermi surface, and instead of the real continuous distribution of gap values uses only two gaps. As shown in Fig. 2(b), let us model the Fermi surface with a sphere and a hyperboloid of revolution with axis parallel to $k_{z}$, and tangent to the sphere as it happens in the real Fermi surface - even though in the 3D BTK model the two Fermi surfaces are treated as if they were completely separated. We also account for the fact that in the Brillouin zone there are 7 cylinders for each sphere.

As for the (uniform) gaps to be associated with the two Fermi surfaces, let us choose the "effective" values $\Delta_{1}=1.3 \mathrm{meV}$ on the spherical Fermi surface and $\Delta_{2}=1.7 \mathrm{meV}$ on the hyperboloid. These values allow reproducing, within the 3D BTK model, the theoretical PCARS spectra that result from Eq. (8) when the real FS and the complete gap distributions are used [36]. For the same reason, we will take $Z_{1}=0.60$ and $Z_{2}=0.95$. Note that the two values of $Z$ refer to the two bands and do not change on changing the direction of current injection. The resulting spectra however show, as in the experiments, a deeper zerobias depletion of the signal in the $c$-axis case, due to the aforementioned "Z-enhancing effect" [13]. The theoretical curves calculated at $T=0.4 \mathrm{~K}$ and with no broadening (i.e., $\Gamma_{1}=\Gamma_{2}=0$ ) are shown in Fig. 2(c). They both show a clear pair of peaks associated with the smaller gap, plus additional peaks (less pronounced in the $a b$-plane direction) associated with the larger gap. The situation is somehow similar to that of $\mathrm{MgB}_{2}$, i.e., the gap residing on the quasi2D Fermi surface sheet (here $\Delta_{1}$ ) is more detectable in the configuration where the projection of the relevant FS has a larger area (i.e., along the $a b$ plane). The experimental situation (Fig. 2(d)) is however much less ideal, since the broadening (not due to the temperature, but probably to pairbreaking effects due to a disordered layer at the interface [37]) actually smears out the double-peak structure and decreases the amplitude of the Andreev signal. Curves very similar to the experimental ones are indeed obtained by introducing in the model the broadening parameters $\Gamma_{1}=0.8 \mathrm{meV}$ and $\Gamma_{2}=0.5 \mathrm{meV}$ (lines in Fig. 4(d)). A comparison of these curves to the experimental ones shows that the position of the peaks is reproduced, as well as the general shape of the curves. The effect of the real continuous distribution of gap values is perceivable in the subgap re- gion, where the experimental curves are more "V-shaped" than the theoretical ones.

\section{Directional PCARS in Co-doped Ca122}

The iron pnictide $\mathrm{CaFe}_{2} \mathrm{As}_{2}$, belonging to the socalled 122 family, becomes superconducting upon application of a modest non-hydrostatic pressure [38] or by partial substitution of Fe with Co [39]. The phase diagram of $\mathrm{Ca}\left(\mathrm{Fe}_{1-x} \mathrm{Co}_{x}\right)_{2} \mathrm{As}_{2}$ as a function of $x[40,41]$ shows that superconductivity sets in abruptly, and with a $T_{C}$ close to the maximum, when the high-temperature magnetic and structural transitions are still present.

Figure 3(a) shows the Fermi surface of 6\% Co-doped $\mathrm{CaFe}_{2} \mathrm{As}_{2}$, calculated within the density-functional theory (DFT) by using the Elk FP-LAPW Code (http://elk.sourceforge.net/) and the GGA approach for the exchange correlation potential. A virtual-crystal approximation was used to account for the partial substitution of Fe with Co [42]. The choice of the doping content is of course based on the fact that the PCARS measurements we are going to discuss were carried out on single crystals of $\mathrm{Ca}\left(\mathrm{Fe}_{1.94} \mathrm{Co}_{0.06}\right)_{2} \mathrm{As}_{2}$ with $T_{C}^{\text {on }}=20 \mathrm{~K}$ and an effective $T_{C}^{\mathrm{eff}}=17 \mathrm{~K}[42]$. Because of the lack of direct experimental information on the low-temperature lattice constants of the compound of our interest, the bandstructure calculations were performed by using the low-temperature lattice constants of the parent compound $\mathrm{CaFe}_{2} \mathrm{As}_{2}$ in the tetragonal phase (estimated from those calculated in the orthorhombic phase $[42,43]$ ) owing to the fact that, at room temperature, the lattice parameters do not change very much upon Co doping [40]. Further details about the calculations can be found in Ref. 42.

A comparison of the Fermi surface (FS) of $6 \%$ Co-doped $\mathrm{CaFe}_{2} \mathrm{As}_{2}$ depicted in Fig. 3(a) with that obtained at slightly smaller and slightly larger Co concentrations shows that at $x=0.06$ the hole-like Fermi surfaces are on the verge of a topological transition, from a warped tubular shape extending through the whole height of the Brillouin zone (BZ), to two separate cup-shaped pockets centered at the $Z$ points. The electron-like FS sheets instead retain their shape of warped cylinders at the corners of the BZ.

To calculate the PCARS spectra within the 3D BTK model, we need to choose a set of analytical surfaces that can be used to mimic the real FS. Figure 3(b) shows a possible model FS (only in the upper half of the Brillouin zone) made up of a one-sheeted hyperboloid of revolution (that simulates the electron-like FS sheets centered in $X$ ) and an oblate spheroid (to simulate the 3D hole-like FS centered in $Z$ ). The proportion between the diameters of the different sheets respects the real one, although the distance between them has been increased for clarity (thus expanding a little the Brillouin zone in the $\left(k_{x}, k_{y}\right)$ plane). To calculate the PCARS spectra within the 3D BTK model, we need to assign a gap to each sheet of the model FS. 
(a)

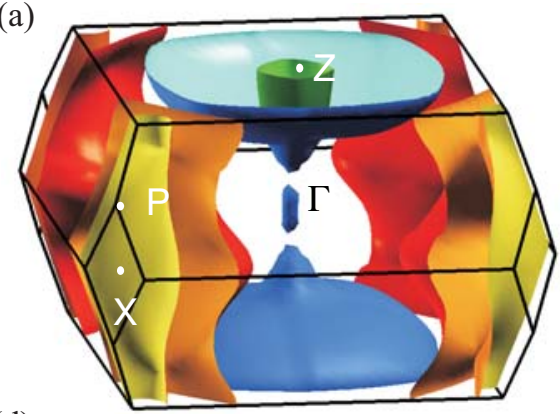

(d)

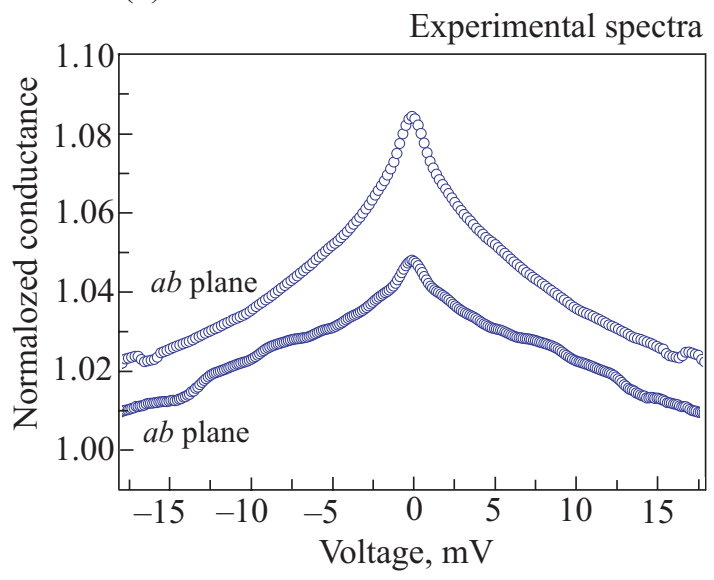

(b)

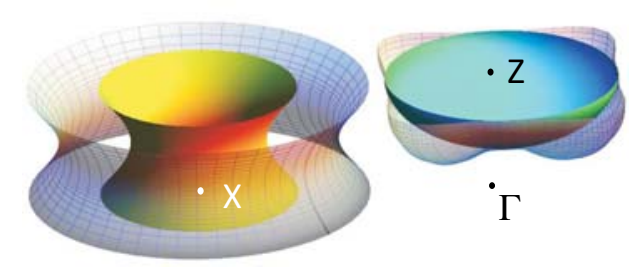

(c)



Fig. 3. (a) Fermi surface of $\mathrm{Ca}\left(\mathrm{Fe}_{1.94} \mathrm{Co}_{0.06}\right)_{2} \mathrm{As}_{2}$. The hole-like FS sheets in the centre of the Brillouin zone is on the verge of a topological transition and is splitting into two closed pockets centered at the $Z$ points. (b) The model Fermi surface used in the 3D BTK model, shown here only in the upper half of the Brillouin zone. Matt surfaces are the Fermi surface sheets, while the gridded surfaces indicate the amplitude of the relevant gap, which is isotropic on the electron-like FS but anisotropic on the hole-like one. (c) Theoretical curves calculated for $I \| a b$ (top) and $I \| c$ (bottom) using the Fermi surface of panel (b) and the gaps indicated in the labels. Here " 1 " and " 2 " refer to the holelike (electron-like) FS, respectively. The other parameters were: for curve $1, \Gamma_{1}=1.25 \mathrm{meV}, Z_{1}=0.05, \Gamma_{2}=6.15 \mathrm{meV}, Z_{2}=0.145$; for curve $2, \Gamma_{1}=1.7 \mathrm{meV}, Z_{1}=0.05, \Gamma_{2}=6.5 \mathrm{meV}, Z_{2}=0.23$. In both cases the angle between the normal to the interface and the $a$ axis was taken to be $\alpha=\pi / 8$. The latter set of parameters was also used to calculate the $c$-axis spectrum (curve 3 ). (d) Two examples of experimental curves measured in single crystals of $6 \%$ Co-doped $\mathrm{CaFe}_{2} \mathrm{As}_{2}$, with the current injected along the $a b$ planes.

In the case of $\mathrm{MgB}_{2}$ there were no doubts about the symmetry of the gaps, which were all isotropic. In the case of Fe-based superconductors, the presence of nodes cannot be excluded a priori - and actually has been predicted to occur in a spin-fluctuation-mediated $s \pm$ picture in particular conditions in 1111 [9] and 122 [10] compounds. In the case of Co-doped $\mathrm{CaFe}_{2} \mathrm{As}_{2}$ there are no specific predictions in this sense; however, there are two orders of reasons that suggest the presence of nodes of some kinds in the gap that resides on the hole-like Fermi surface.

The first reason is experimental. In this compound, $100 \%$ of the conductance curves (measured with $I \| a b$ ) show a zero-bias peak and broad shoulders at a finite energy (5-6 meV) as shown for example in Fig. 3(d). There are various phenomena that can give rise to a zero-bias peak in point-contact spectroscopy [3], but there are good reasons [42] to think that, in this case, its origin is intrinsic and is not, e.g., related to experimental artifacts. Indeed, a zerobias maximum or peak is observed when the gap has lines of either nodes (i.e., separating regions of positive and negative order parameter) or zeros [6] somewhere on the Fermi surface. As shown elsewhere [13] if there is a large broadening, as in our case (the amplitude of the curves of Fig. 3(d) is rather small) it becomes virtually impossible to discriminate between these two alternatives.

The second reason is that the emergence of lines of nodes (that are vertical in the proximity of the top and bottom of the BZ) on the outer hole-like FS has been predicted in Ba122 $[10,11]$ in particular conditions, i.e., when the pnictogen height $z_{\mathrm{As}}$ is reduced, for example by isovalent $\mathrm{P}$ doping [10]. This is accompanied by a growth in the size of the outer hole-like FS around the $Z$ points, a phenomenon which occurs also in $\mathrm{Ca}\left(\mathrm{Fe}_{1-x} \mathrm{Co}_{x}\right)_{2} \mathrm{As}_{2}$ at the increase of $x$. At $x=0.06$ the hole-like Fermi surface even becomes completely $3 \mathrm{D}$ by splitting into closed pockets; a possible symmetry of the gap on these surfaces can thus be imagined to be an evolution of the peculiar 3D nodal symmetry (with vertical node lines) in these conditions. We guess that the result of 
this evolution can be expressed as a 3D fully anisotropic swave symmetry, whose expression in the reciprocal space is $\Delta_{1}(\theta, \phi)=\Delta_{1} \cos ^{4} 2 \theta \sin ^{2} \phi$ (here, the origin is placed in the center of the relevant FS sheet). This gap does not show nodes (i.e., it does not change sign) but only lines of zeros that intersect each other above and below the $Z$ points. The choice of this symmetry may seem unreasonable given that a sign change of the order parameter is predicted in Refs. 10, 11. Indeed, another possibility is to use a $3 \mathrm{D} d$-wave symmetry $\Delta_{1}(\theta, \phi)=\Delta_{1} \cos 2 \theta \sin ^{2} \phi$ [42] but: i) the results do not change very much once a large broadening is inserted in the BTK model [13]; ii) the symmetry of the gap of Refs. 10,11 in the horizontal plane through $Z$ is different from the $d$ wave one and features two additional axes of equation $k_{y}= \pm k_{x}$; in these conditions, interference effects between HLQ and ELQ are considerably reduced and the zero-bias maximum is expected to arise mostly from the fact that the gap is very small in some angular regions. The amplitude and the spatial modulation of this gap is shown as a gridded surface around the hole pocket in Fig. 3(b). The gap on the electron-like FS is instead chosen to be isotropic.

Figure 3(c) shows two examples of $a b$-plane PCARS spectra (curves 1 and 2) calculated at $T=2.2 \mathrm{~K}$ (the lowest experimental temperature in this case) within the $3 \mathrm{D}$ BTK model, by using values of the gaps that allow reproducing the experimental $a b$-plane curves of Fig. 3(d). The gap amplitudes are indicated in the label, where " 1 " refers to the hole-like FS and "2" to the electron-like one; the other best-fitting parameters are listed in the caption. Due to the very small amplitude of the curves, a large broadening was necessary. The parameters that generate curve 2 were also used to calculate a theoretical $c$-axis spectrum (curve 3) even though in this case we do not have any experimental curve in this configuration. The success of the model in reproducing the experimental curves suggests that the chosen gap symmetry is probably a good approximation to the real one. Moreover, as pointed out elsewhere [42], the presence of zero-bias maxima in the $c$-axis conductance necessarily requires that the FS hosting the anisotropic gap is a closed surface, as it results from bandstructure calculations.

\section{Directional PCARS in Co-doped Ba122}

The iron arsenide $\mathrm{BaFe}_{2} \mathrm{As}_{2}$ is probably the most widely studied representative of the so-called 122 family. One of the reasons is that it can be made superconducting by hole doping (e.g., by $\mathrm{K}$ doping in the $\mathrm{Ba}$ site), isoelectronic doping (e.g., by partial substitution of $\mathrm{Ba}$ with $\mathrm{Sr}$, As with $\mathrm{P}$ or Fe with $\mathrm{Ru}$ ), electron doping (by Co substitution in the Fe site) or external pressure, giving rise to a variety of phase diagrams that can be in principle compared to extract information about the mechanisms of superconductivity [44]. The second reason is that single crystals of all these compounds can be grown large enough to allow an extensive experimental investigation by means of almost all the available techniques (including, for example, angle-resolved photoemission spectroscopy). Apart from minor discrepancies (mainly about the doping contents) the phase diagrams of $\mathrm{Ba}\left(\mathrm{Fe}_{1-\chi} \mathrm{Co}_{\chi}\right)_{2} \mathrm{As}_{2}$ indicate the existence of a wide, well-defined and asymmetric superconducting dome extending from $x \simeq 0.03$ to $x \simeq 0.15$ [45], with a maximum at $x \simeq 0.07$. The lines indicating the tetragonal-toorthorhombic structural transition and the magnetic transition intersect the superconducting dome in the proximity of the maximum; in the "underdoped" region, experiments seem to indicate microscopic coexistence of SDW and superconducting states [45]. Figure 4(a) shows the Fermi surface of $\mathrm{Ba}\left(\mathrm{Fe}_{1-x} \mathrm{Co}_{x}\right)_{2} \mathrm{As}_{2}$ at $x=0.08$ calculated in the same way as in Co-doped Ca122, by using the lattice constants $a=b=3.9625 \AA$ and $c=13.0168 \AA$ as in Ref. 46 . The Fermi surface features two hole-like sheets around the $\Gamma-Z$ axis - both having the shape of warped cylinders, although the warping of the outer one is much more marked - and two electron-like warped cylinders with the characteristic "elliptical" cross-section that depends on $k_{z}$. Figure 4(b) shows the model Fermi surface we chose for the calculation of the PCARS spectra within the 3D BTK model. It is simply made up of two hyperboloids of revolution, one for the outer hole-like FS sheet (the inner one is neglected) and one for the two electron-like sheets. The problem now arises of which symmetry of the order parameter one should assume to calculate the PCARS spectra.

A number of experimental results reported in literature (including ARPES [47]) indicate for $\mathrm{Ba}\left(\mathrm{Fe}_{1-x} \mathrm{Co}_{x}\right)_{2} \mathrm{As}_{2}$ two isotropic gaps. The first directional PCARS measurements in single crystals $\left(x=0.07, T_{c}=23 \mathrm{~K}\right)$ [48] that were carried out in the needle-anvil configuration and with the current along the $c$ axis, showed clear Andreev signals but no evidence of multiple gaps and were fitted by a single-gap, s-wave 2D BTK model. Our directional PCARS measurements in nominally $10 \%$ Co-doped Ba122 single crystals with $T_{C}=24.5 \mathrm{~K}$ [49] gave spectra with clear two-band structures in either the $I \| a b$ and $I \| c$ configurations. These spectra were successfully fitted by using a two-band 2D BTK model with two isotropic gaps.

On the other hand, indications in favor of a complicated 3D structure of the gap (at least on the hole-like FS) with lines of zeros or deep minima were provided by directional thermal conductivity [50] and Raman spectroscopy [51] measurements; this possibility has been shown theoretically to be compatible with the general $s \pm$ symmetry [12].

Based on the above, here we will first calculate the theoretical PCARS spectra within the 3D BTK model by assuming two isotropic gaps, $\Delta_{1}$ on the hole-like FS and $\Delta_{2}$ on the electron-like FS, and we will see whether this assumption allows reproducing the experimental data, in particular the representative spectra reported in Fig. 4(d). The first three spectra were measured in single crystals of $\mathrm{Ba}\left(\mathrm{Fe}_{1-x} \mathrm{Co}_{x}\right)_{2} \mathrm{As}_{2}$ with nominal $x=0.1$, bulk $T_{c}^{\text {on }}=$ $=24.5 \mathrm{~K}$ and $\delta T_{C}=1 \mathrm{~K}$ [49], with the current parallel to 
(a)

(d)
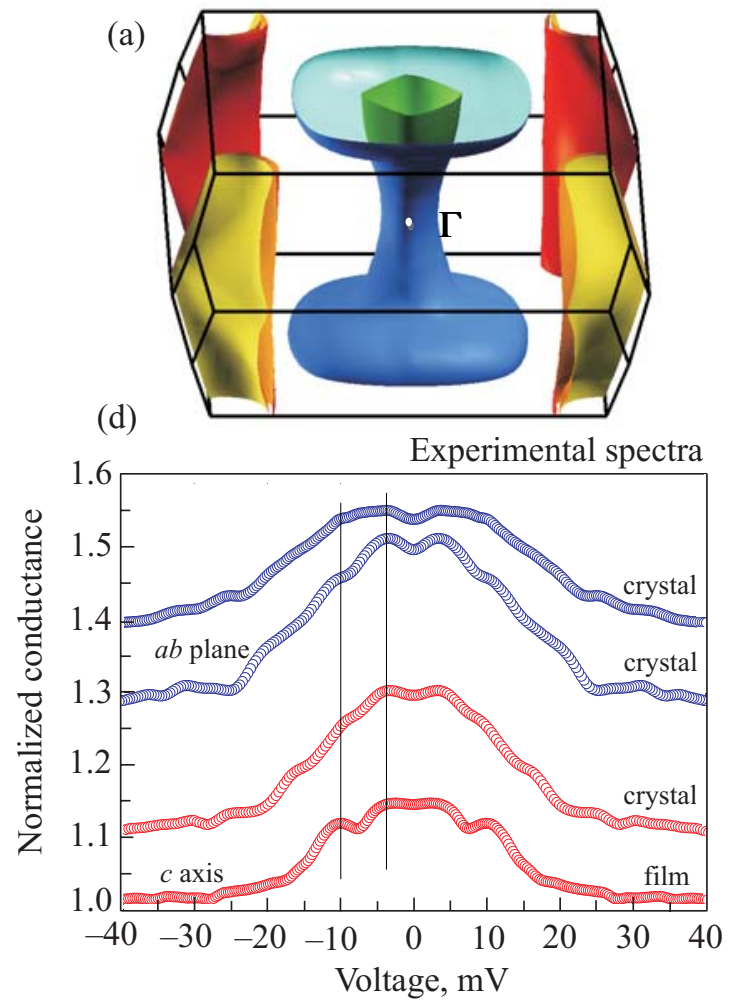

(b)

(c)
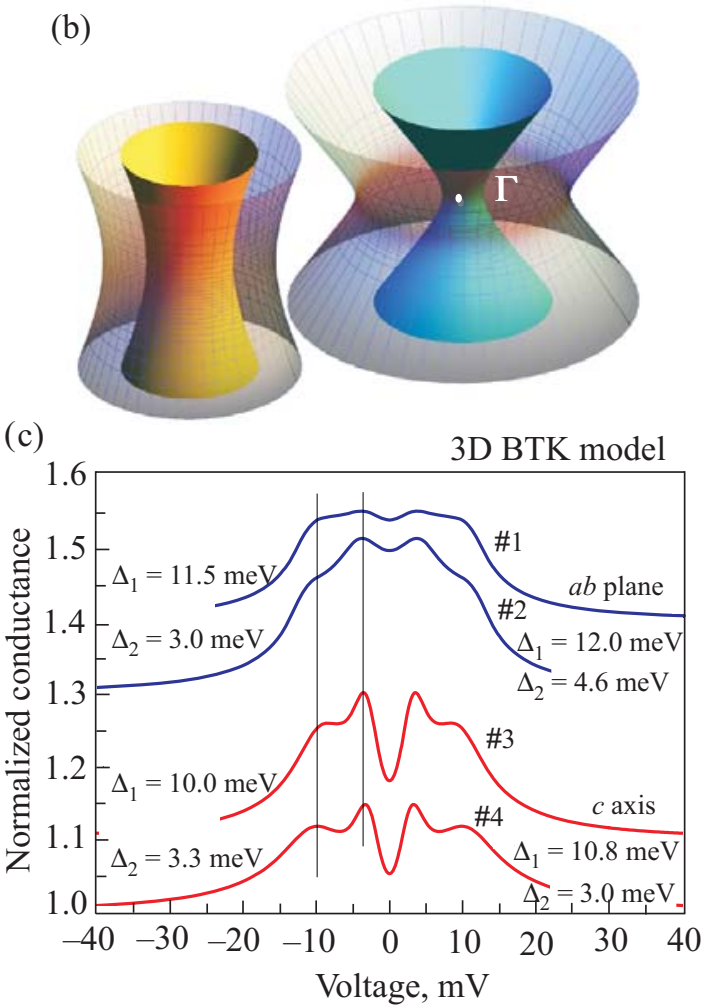

Fig. 4. (a) Fermi surface of $\mathrm{Ba}\left(\mathrm{Fe}_{1.92} \mathrm{Co}_{0.08}\right)_{2} \mathrm{As}_{2}$, with the strongly warped hole-like $\mathrm{FS}$ sheets around $\Gamma$ and the electron-like $\mathrm{FS}$ sheets at the corners of the Brillouin zone. (b) The model Fermi surface used in the 3D BTK model. Matt surfaces are the Fermi surface sheets, while the gridded surfaces indicate the amplitude of the relevant gap. The drawing refers to the case of isotropic gaps on both the bands. (c) Theoretical curves calculated for $I \| a b$ (1 and 2) and $I \| c$ (3 and 4) and using the Fermi surface of panel (b). The gap amplitudes indicated in the labels were chosen in order to fit the position of the gap features in the experimental curves of panel (d). The other fitting parameters are the following: for curve $1, \Gamma_{1}=1.85 \mathrm{meV}, Z_{1}=0.03, \Gamma_{2}=3.6 \mathrm{meV}, Z_{2}=0.31$; for curve $2, \Gamma_{1}=1.75 \mathrm{meV}, Z_{1}=0.08$, $\Gamma_{2}==3.0 \mathrm{meV}, Z_{2}=0.245$; for curve $3, \Gamma_{1}=2.8 \mathrm{meV}, Z_{1}=0.05, \Gamma_{2}=1.3 \mathrm{meV}, Z_{2}=0$; for curve $4, \Gamma_{1}=3.85 \mathrm{meV}, Z_{1}=0.1, \Gamma_{2}=1.4$ $\mathrm{meV}, Z_{2}=0$. (d) Some examples of the experimental curves measured in $8 \%$ Co-doped $\mathrm{BaFe}_{2} \mathrm{As}_{2}$ with $I \| a b$ (top) and $I \| c$ (bottom). The lowest-lying curve was measured in epitaxial films [52] with $x=0.08$ and $T_{C}=(23.8 \pm 0.7) \mathrm{K}$, the others in single crystals.

the $a b$ plane (first two curves) and to the $c$ axis (third curve). All of them feature multiple structures that, as we showed elsewhere [49] can be interpreted as being due to two distinct gaps and to the strong coupling of electrons to spin fluctuations whose characteristic energy $\Omega$ is around $12 \mathrm{meV}$ (the relevant structure occurring at $\Delta_{1}+\Omega$ if $\Delta_{1}$ is the large gap). The bottom curve of Fig. 4(d) was measured in epitaxial thin films (having the $c$ axis normal to the surface) with $x=0.08$ and $T_{c}=(23.8 \pm 0.7) \mathrm{K}$ grown on a $\mathrm{MgO}$ substrate, with a Fe buffer layer. The multigap structures in this curve are even clearer than in single crystals; as shown by the vertical lines, the position of the gap features is the same for all the curves, which is a good indication of consistency.

By using the 3D BTK model and the Fermi surface of panel (b), we can then try to fit the experimental curves of panel (d). To do so, we will take all the parameters (including the gap amplitudes) as being adjustable, rather than fixing the values of the gaps as we did in $\mathrm{CaC}_{6}$ or $\mathrm{MgB}_{2}$. Figure 4(c) reports the theoretical curves that best fit the experimental ones of panel (d). The gap amplitudes are reported in the legend, the other parameters in the caption. It is interesting to note that: i) the fit is generally capable of reproducing the position of the gap-related structures by using consistent values of the gaps, i.e., $\Delta_{1}=(11.0 \pm 1.0)$ $\mathrm{meV}$ and $\Delta_{2}=(3.8 \pm 0.8) \mathrm{meV}$; ii) the fit in the region $\mathrm{eV}>\Delta_{1}$ works well in thin films, but fails in single crystals where large electron-boson coupling structures dominate the spectrum above $15 \mathrm{meV}$. This is actually obvious since the electron-boson structures cannot be reproduced by a BTK model unless the explicit energy dependence of the order parameter is taken into account, as we did in Refs. 49 and 13; iii) the fit works well in the subgap region $e V<\Delta_{1}$ only in $a b$-plane contacts. In the case $I \| c$ instead the theoretical curves systematically show a zero-bias dip which is much deeper than the experimental one. This 
happens even if both $Z_{1}$ and $Z_{2}$ are unrealistically taken to be zero, because of the aforementioned Z-enhancing effect related to the shape of the relevant FS sheet.

The failure of the model in reproducing the zero-bias region of the curves in $c$-axis contacts clearly indicates that either: i) there is some additional effect, not considered here, that gives rise to a zero-bias enhancing that partially compensates the signal depletion due to the shape of the Fermi surface, or ii) the smaller gap is not isotropic. The two possibilities are still under investigation, but preliminary (although detailed) results show that the existence on the hole-like FS of horizontal and/or vertical node lines could not be sufficient to explain the observed discrepancy. In this regard, it is worth noting that only the use of the 3D BTK model allows unveiling effects like this, while the 2D BTK model (based on the assumption of spherical Fermi surfaces and with an adjustable weight) allows fitting the curves in both directions without any difficulty, as we did in Ref. 49.

\section{Conclusions}

The discovery of new multiband and anisotropic superconductors at the beginning of the third millennium has revitalized the research about superconductivity, but has also required a refinement and a development of the experimental and theoretical techniques. With the progress in the investigation of these compounds, the necessity has arisen to go deeper and deeper in the subtle details of their properties in order to discriminate between different possible pairing mechanisms, understand how general some properties are, determine which of them are fundamental and which are sample-dependent, and so on.

Point-contact Andreev-reflection spectroscopy is a simple, versatile and very powerful tool for the investigation of superconductors. However, the interpretation of the PCARS results in multiband and/or anisotropic compounds is much more challenging than it used to be in conventional superconductors and possibly even in cuprates. In particular, although the use of the simplest models for the fit of the PCARS spectra can usually provide good general information about the number and amplitude of the gaps, more sophisticated methods of analysis are required to study the fine details of the gap symmetry and structure.

In this review we have shown that the capabilities of PCARS can be extended - though without any particular improvement in the experimental technique in itself - by suitably generalizing the Blonder-Tinkham-Klapwijk model to the case of multiple Fermi surface sheets and anisotropic order parameters, thanks to a systematic link with Fermi-surface calculations. The so-called "3D" BTK model makes use of a model FS that mimics the real one, and can account for virtually any kind of $k$ dependence of the order parameter. With respect to the $1 \mathrm{D}$ and $2 \mathrm{D}$ versions of the same model, it relaxes some simplifying as- sumptions that are no longer justified in these compounds; moreover, the "weight" of the different bands in the total conductance for any given direction of current injection is no longer an adjustable parameter but is completely determined by the barrier height and by the shape of the Fermi surface. With respect to the full integration over the real FS, it has the advantage of being analytical and thus much less demanding in terms of calculation.

To show the benefits of using this approach, we have reported some representative examples of its application to multiband or anisotropic superconductors. In $\mathrm{MgB}_{2}$, the paradigm of multiband superconductivity, we have shown that the 3D BTK model allows reproducing very well the directional PCARS spectra by using the values of the (isotropic) gaps calculated from first principles [7,9]. Also the systematic zero-bias depletion of conductance observed in $c$-axis contacts is naturally reproduced as a consequence of the different dimensionality of the $\sigma$ and $\pi$ bands. In $\mathrm{CaC}_{6}$, an anisotropic superconductor with a marked $\mathbf{k}$ dependence of the order parameter but no lines of nodes [33], we have shown that theoretical PCARS spectra in excellent agreement with the experimental ones can be obtained by using an effective model with two uniform gaps associated to two inequivalent FS sheets having different dimensionality. In this case, the availability of the complete map of gap amplitude on the Fermi surface [33] allows calculating the PCARS spectra also by integrating explicitly over the real FS [36]; the comparison of the spectra however shows that the effective "two-gap, two-FS" model is sufficient to perfectly catch the features of the system. In Co-doped $\mathrm{CaFe}_{2} \mathrm{As}_{2}$, the systematic observation of zero-bias maxima in the PCARS spectra suggests the presence of lines of nodes crossing the Fermi surface [42]; however, a reasonable guess about the shape of these nodes and their location on the FS sheets can be made only by studying the FS geometry and exploiting theoretical predictions about the emergence of nodes in similar compounds $[10,11]$. By using a nodeless large gap on the electron 2D-like FS and a smaller gap with full 3D anisotropy on the hole 3D-like pockets, we have shown that it is possible to reproduce rather well the experimental $a b$-plane spectra and to predict the shape of $c$-axis ones. Finally, in Co-doped $\mathrm{CaFe}_{2} \mathrm{As}_{2}$ the experimental PCARS spectra do not show any zero-bias peak and can indeed be fitted within the $2 \mathrm{D}$ BTK model by using two isotropic gaps. However, if the geometry of the FS is taken into account, as in the 3D model, one discovers that two isotropic gaps cannot account completely for the experimental spectra. In particular, a deep zero-bias dip for $I \| c$ is expected but not observed. This suggests that either the gap is zero in some regions of the FS (although simple node lines seem to be insufficient, which could point to the existence of "hot spots" recently predicted on the hole-like FS), or additional phenomena are taking place that give rise to a zero-bias peak that partly compensates the depletion. 


\section{Acknowledgments}

We acknowledge the financial support of the European Community through to the Collaborative EU-Japan Project "IRON SEA" (NMP3-SL-2011-283141), and of the Italian Ministry of Research through the PRIN Project N. 2008XWLWF9-005. Our warmest thanks to all the people who contributed to the research summarized in this paper: G.A. Ummarino, S. Galasso, G. Profeta for help in theoretical analysis and bandstructure calculations; J. Karpinski, N.D. Zhigadlo, Z. Bukowski, J. Jiang, J.S. Kim, K. Iida and F. Kurth for providing us with crystals and films used for PCARS measurements. RSG acknowledges R.K. Kremer and the Max Planck Institute for Solid State Research in Stuttgart, where the measurements on $\mathrm{Ca}(\mathrm{Fe}, \mathrm{Co})_{2} \mathrm{As}_{2}$ were carried out.

1. I.K. Yanson, Sov. Phys. JETP 39, 506 (1974).

2. A.M. Duif, A.G.M. Jansen, and P. Wyder, J. Phys.: Condens. Matter 1, 3157 (1989).

3. Y.G. Naidyuk and I.K. Yanson, Point-Contact Spectroscopy, Springer (2005).

4. D. Saint-James, J. Phys. 25, 899 (1964).

5. G.E. Blonder, M. Tinkham, and T.M. Klapwijk, Phys. Rev. B 25, 4515 (1982).

6. S. Kashiwaya, Y. Tanaka, M. Koyanagi, and K. Kajimura, Phys. Rev. B 53, 2667 (1996).

7. A. Brinkman, A.A. Golubov, H. Rogalla, O.V. Dolgov, J. Kortus, Y. Kong, O. Jepsen, and O. K. Andersen, Phys. Rev. B 65, 180517 (2002).

8. D. Daghero and R. Gonnelli, Supercond. Sci. Technol. 23, 043001 (2010).

9. K. Kuroki, H. Usui, S. Onari, R. Arita, and H. Aoki, Phys. Rev. B 79, 224511 (2009).

10. K. Suzuki, H. Usui, and K. Kuroki, J. Phys. Soc. Jpn. 80, 013710 (2011).

11. S. Graser, A.F. Kemper, T.A. Maier, H.-P. Cheng, P.J. Hirschfeld, and D.J. Scalapino, Phys. Rev. B 81, 21450 (2010).

12. I.I. Mazin, T.P. Devereaux, R. Hackl, B. Muschler, J.G. Analytis, J.-H. Chu, and I.R. Fisher, Phys. Rev. B 82, 180502(R) (2010).

13. D. Daghero, M. Tortello, G. Ummarino, and R.S. Gonnelli, Rep. Prog. Phys. 74, 124509 (2011).

14. A. Plecenik, M. Grajcar, v. Beňačka, P. Seidel, and A. Pfuch, Phys. Rev. B 49, 10016 (1994).

15. I.I. Mazin, Phys. Rev. Lett. 83, 1427 (1999).

16. Y. Kong, O.V. Dolgov, O. Jepsen, and O.K. Andersen, Phys. Rev. B 64, 020501(R) (2001).

17. A.Y. Liu, I.I. Mazin, and J. Kortus, Phys. Rev. Lett. 87, 87005 (2001).

18. C. Buzea and T. Yamashita, Supercond. Sci. Technol. 14, R115 (2001).

19. H.J. Choi, D. Roundy, H. Sun, M.L. Cohen, and S.G. Louie, Nature 418, 758 (2002).
20. P. Szabó, P. Samuely, J. Kacmarcik, T. Klein, J. Marcus, D. Fruchart, S. Miraglia, C. Marcenat, and A.G.M. Jansen, Phys. Rev. Lett. 87, 137005 (2001).

21. Y. Bugoslavsky, Y. Miyoshi, G.K. Perkins, A.V. Berenov, Z. Lockman, J.L. MacManus-Driscoll, L.F. Cohen, A.D. Caplin, H.Y. Zhai, M.P. Paranthaman, H.M. Christen, and M. Blamire, Supercond. Sci. Technol. 15, 526 (2002).

22. Y.G. Naidyuk, I.K. Yanson, L. Tyutrina, N.L. Bobrov, P.N. Chobov, W.N. Kang, H.J. Kim, E.M. Choi, and S.I. Lee, JETP Lett. 75, 283 (2002).

23. I.K. Yanson and Y.G. Naidyuk, Fiz. Nizk. Temp. 30, 355 (2004) [Low Temp. Phys. 30, 261 (2004)].

24. M. Iavarone, G. Karapetrov, A.E. Koshelev, W.K. Kwok, G.W. Crabtree, D.G. Hinks, W.N. Kang, E.-M. Choi, H.J. Kim, H.-J. Kim, and S.I. Lee, Phys. Rev. Lett. 89, 187002 (2002).

25. Y.G. Naidyuk, I.K. Yanson, O.E. Kvitnitskaya, S. Lee, and S. Tajima, Phys. Rev. Lett. 90, 197001 (2003).

26. I.K. Yanson, V.V. Fisun, N.L. Bobrov, Y.G. Naidyuk, W.N. Kang, E.-M. Choi, H.-J. Kim, and S.-I. Lee, Phys. Rev. B 67, 024517 (2003).

27. R.S. Gonnelli, D. Daghero, G.A. Ummarino, V.A. Stepanov, J. Jun, S.M. Kazakov, and J. Karpinski, Phys. Rev. Lett. 89, 247004 (2002).

28. T. Dahm and N. Schopohl, Phys. Rev. Lett. 91, 017001 (2003).

29. N. Emery, C. Hérold, M. d'Astuto, V. Garcia, C. Bellin, J. Marêchré, P. Lagrange, and G. Loupias, Phys. Rev. Lett. 95, 087003 (2005).

30. I.I. Mazin, L. Boeri, O.V. Dolgov, A.A. Golubov, G.B. Bachelet, M. Giantomassi, and O.K. Andersen, Physica C 460-462, 116 (2007).

31. M. Calandra and F. Mauri, Phys. Rev. Lett. 95, 237002 (2005).

32. J.S. Kim, L. Boeri, R.K. Kremer, and F.S. Razavi, Phys. Rev. B 74, 214513 (2006).

33. A. Sanna, G. Profeta, A. Floris, A. Marini, E.K.U. Gross, and S. Massidda, Phys. Rev. B 75, 020511(R) (2007).

34. N. Bergeal, V. Dubost, Y. Noat, W. Sacks, D. Roditchev, N. Emery, C. Hrerold, J.-F. Marêchré, P. Lagrange, and G. Loupias, Phys. Rev. Lett. 97, 077003 (2006).

35. C. Kurter, L. Ozyuzer, D. Mazur, J.F. Zasadzinski, D. Rosenmann, H. Claus, D.G. Hinks, and K.E. Gray, Phys. Rev. B 76, 220502 (2007).

36. R.S. Gonnelli, D. Daghero, D. Delaude, M. Tortello, G.A. Ummarino, V.A. Stepanov, J.S. Kim, R.K. Kremer, A. Sanna, G. Profeta, and S. Massidda, Phys. Rev. Lett. 100, 207004 (2008).

37. P. Chalsani, S.K. Upadhyay, O. Ozatay, and R. Burham, Phys. Rev. B 75, 094417 (2007).

38. S. Torikachvili, S.L. Budko, N. Ni, and P.C. Canfield, Phys. Rev. Lett. 101, 057006 (2008).

39. M. Matusiak, Z. Bukowski, and J. Karpinski, Phys. Rev. B 81, 020510(R) (2010).

40. R. Hu, S. Ran, S. Bud'ko, W.E. Straszheim, and P.C. Canfield, Philos. Mag. 92, 3113 (2012). 
41. L. Harnagea, S. Singh, G. Friemel, N. Leps, D. Bombor, M. Abdel-Hafiez, A.U.B. Wolter, C. Hess, R. Klingeler, G. Behr, S. Wurmehl, and B. Büchner, Phys. Rev. B 83, 094523 (2011).

42. R.S. Gonnelli, M. Tortello, D. Daghero, R.K. Kremer, Z. Bukovski, N.D. Zhigadlo, and J. Karpinski, Supercond. Sci. Technol. 25, 065007 (2012).

43. N. Colonna, G. Profeta, A. Continenza, and S. Massidda, Phys. Rev. B 83, 094529 (2011).

44. J. Paglione and R.L. Greene, Nature Phys. 6, 645 (2010).

45. M.D. Lumsden and A.D. Christianson, J. Phys.: Condens. Matter 22, 203203 (2010).

46. D.J. Singh, Phys. Rev. B 78, 094511 (2008).

47. K. Terashima, J.H. Bowen, K. Nakayama, T. Sato, P. Richard, Y.-M. Xu, L.J. Li, G.H. Cao, Z.-A. Xu, H. Ding, and T. Takahashi, PNAS (USA) 106, 7330 (2009).
48. P. Samuely, Z. Pribulová, P. Szabó, G. Pristráš, S.L. Bud'ko, and P.C. Canfield, Physica C 469, 507 (2009).

49. M. Tortello, D. Daghero, G.A. Ummarino, V.A. Stepanov, J. Jiang, J.D. Weiss, E.E. Hellstrom, and R.S. Gonnelli, Phys. Rev. Lett. 105, 237002 (2010).

50. J.-P. Reid, M.A. Tanatar, X.G. Luo, H. Shakeripour, N. Doiron-Leyraud, N. Ni, S.L. Budko, P.C. Canfield, R. Prozorov, and L. Taillefer, Phys. Rev. B 82, 064501 (2010).

51. B. Muschler, W. Prestel, R. Hackl, T.P. Devereaux, J.G. Analytis, J.-H. Chu, and I.R. Fisher, Phys. Rev. B 80, 180510(R) (2009).

52. K. Iida, J. Hänisch, S. Trommler, S. Haindl, F. Kurth, R. Hühne, L. Schultz, and B. Holzapfel, Supercond. Sci. Technol. 24, 125009 (2011). 